\title{
FERTILIZER RECOMMENDATION FOR FOUR CROP BASED CROPPING PATTERN: POTATO- BORO- T. AUS-T. AMAN UNDER AEZ - 11
}

\author{
A. Barman ${ }^{1 *}$, S. Shome ${ }^{2}$, M.J. Alam ${ }^{3}$, S. Akhter ${ }^{1}$ and M.A. Hossain ${ }^{1}$ \\ ${ }^{1}$ Soil Science Division, BARI, Gazipur, Bangladesh \\ ${ }^{2}$ Department of Agronomy, SAU, Dhaka, Bangladesh \\ ${ }^{3}$ RARS, BARI, Jashore, Bangladesh \\ *Corresponding E-mail: alakbarman.sau@gmail.com \\ (Received: 19 May 2019, Accepted: 30 August 2019)
}

Keywords: Cropping pattern, fertilizer, rice equivalent yield

\begin{abstract}
A field experiment on Potato-Boro-T. Aus-T. Aman cropping pattern was conducted in the High Ganges Floodplain Soil of Jashore (AEZ - 11) during 2014-2015 and 2015-2016 to find out optimum fertilizer management for intensive cropping pattern, in relation to soil health.There were eight different treatments viz. $\mathrm{T}_{1}=100 \%$ NPKSZnB ( $\mathrm{STB}=$ Soil Test Based), $\mathrm{T}_{2}=\mathrm{T}_{1}+25 \% \mathrm{~N}, \mathrm{~T}_{3}=\mathrm{T}_{1}+25 \% \mathrm{NP}, \mathrm{T}_{4}=\mathrm{T}_{1}+$ $25 \% \mathrm{NK}, \mathrm{T}_{5}=\mathrm{T}_{1}+25 \% \mathrm{PK}, \mathrm{T}_{6}=\mathrm{T}_{1}+25 \% \mathrm{NPK}, \mathrm{T}_{7}=75 \%$ of $\mathrm{T}_{1}$, and $\mathrm{T}_{8}=$ Native fertility (control) as individual crop management. The experiment was laid out in randomized complete block design with three replications. Results revealed that the tuber yield of potato, grain yields of boro, T. Aus and T. Aman were significantly influenced by the fertilizer treatments. The maximum tuber yield $\left(25.70\right.$ and $\left.24.80 \mathrm{t} \mathrm{ha}^{-1}\right)$, grain yields of boro $\left(6.50\right.$ and $\left.6.31 \mathrm{t} \mathrm{ha}^{-1}\right)$, T. Aus $\left(3.13\right.$ and $\left.3.10 \mathrm{t} \mathrm{ha}{ }^{-1}\right)$ and $\mathrm{T}$. Aman (3.95 and $3.98 \mathrm{t} \mathrm{ha}^{-1}$ ) was obtained from the $\mathrm{T}_{6}$ treatment where 25\% additional NPK was added with 100\% STB in both the year. These yields were statistically similar with that produced by all other fertilizer treatments except the native fertility treatment. Highest rice equivalent yield (REY) of $12.63 \mathrm{t} \mathrm{ha}^{-1}$ was obtained from $\mathrm{T}_{6}$ treatment whereas lowest REY of $5.93 \mathrm{t} \mathrm{ha}^{-1}$ was obtained from control.
\end{abstract}

\section{Introduction}

Feeding the enormous population of Bangladesh is a great challenge for the farmers and agricultural scientists. At present the total cultivable land of Bangladesh is 8.5 million hectare which is decreasing gradually because cultivable land is used as non-agriculture (BBS, 2012). The present cropping intensity is $190 \%$ and that can be increased up to some extent by improving the present cropping pattern, incorporating short duration crops and through management of cultivation practices (ORC, 2013). Most of the major cropping patterns practiced around the country are comprised of two to three crops a year. Recently four crop based cropping pattern has been introduced in many AEZs.

But intensive land use without appropriate soil management has caused depletion of soil fertility in Bangladesh. The present system of fertilizer application is mostly based on the nutrient requirement of individual crops ignoring the carryover effect of the organic or inorganic fertilizers applied to the preceding crop. For maintaining soil quality and attainable crop yields for such intensive cropping pattern, proper fertilizer management is required. Considering the above facts, the present study was undertaken to find out judicious fertilizer recommendation for Potato- Boro-T. Aus- T. Aman cropping pattern for AEZ-11. 


\section{Materials and Methods}

The field experiment was conducted at the central farm of RARS Jashore (AEZ 11) during 2014-2015 and 2015-2016. The initial soil samples, collected before establishing the experiment from a depth of $0-15 \mathrm{~cm}$ were analyzed in the laboratory following standard methods. Initial values of some important soil chemical parameters of the experimental soil are presented in Table 1.

Table 1. Chemical properties of experimental soil (initial) at RARS Jashore

\begin{tabular}{|c|c|c|c|c|c|c|c|c|c|c|c|c|}
\hline \multirow[b]{2}{*}{$\mathrm{pH}$} & $\mathrm{OM}$ & $\mathrm{Ca}$ & $\mathrm{Mg}$ & $\mathrm{K}$ & \multirow{2}{*}{$\begin{array}{l}\text { Total } \\
\mathrm{N} \%\end{array}$} & $\mathrm{P}$ & $\mathrm{S}$ & B & $\mathrm{Cu}$ & $\mathrm{Fe}$ & $\mathrm{Mn}$ & $\mathrm{Zn}$ \\
\hline & (\%) & & 100 & soil & & \multicolumn{7}{|c|}{$\mu \mathrm{g} \mathrm{g}{ }^{-1}$} \\
\hline 7.7 & 0.92 & 7.8 & 3.4 & 0.18 & 0.048 & 15 & 13 & 0.14 & 3 & 29 & 5 & 1.8 \\
\hline Criti & level & 2.0 & 0.5 & 0.12 & 0.12 & 10 & 10 & 0.2 & 0.2 & 4 & 1 & 0.6 \\
\hline
\end{tabular}

The experiment was laid out in a randomized complete block design with three replications. Eight different treatments viz. $\mathrm{T}_{1}=100 \% \mathrm{STB}, \mathrm{T}_{2}=\mathrm{T}_{1}+25 \% \mathrm{~N}, \mathrm{~T}_{3}$ $=\mathrm{T}_{1}+25 \% \mathrm{NP}, \quad \mathrm{T}_{4}=\mathrm{T}_{1}+25 \% \mathrm{NK}, \mathrm{T}_{5}=\mathrm{T}_{1}+25 \% \mathrm{PK}, \quad \mathrm{T}_{6}=\mathrm{T}_{1}+25 \% \mathrm{NPK}$, $\mathrm{T}_{7}=75 \%$ of $\mathrm{T}_{1}, \mathrm{~T}_{8}=$ Native fertility was selected for different plots randomly as individual crop management. The unit plot size was $2.5 \mathrm{~m} \times 3.0 \mathrm{~m}$. Potato (var. Diamant) was used as test crop for the first component of the pattern. Potato seeds were a spacing of $60 \times 25 \mathrm{~cm}$ on November 11, 2014 and November 10, 2015. Fertilizer N-P-K-S-Zn and B were supplied from urea, TSP, MP, Gypsum, Zinc sulphate and Boric acid respectively. All P K S Zn B and 1/2 of $\mathrm{N}$ were applied at the time of final land preparation. The remaining half of $\mathrm{N}$ was applied as top dress at 30-35 days after sowing. Two irrigations and other intercultural operations were done as and when required.

The potato was harvested on January 29, 2015 and January 30, 2016. Data on yield and yield contributing characters of potato were recorded and statistically analyzed.

After potato, Boro rice var. BRRI dhan28 was transplanted on February 3, 2015 and February 2, 2016 maintaining $20 \times 15 \mathrm{~cm}$ spacing. All fertilizers including $1 / 3^{\text {rd }}$ of $N$ were applied before transplanting. Rest of $N$ was applied in two installments at 15 and 45 days after transplanting. Boro rice was harvested on 12 May, 2015 and 14 May, 2016. For T. Aus rice, var. BRRI dhan48 was used transplanting was done on 15 May 2015 and 17 may 2016 maintaining $20 \times 15 \mathrm{~cm}$ plant spacing. All fertilizers including $1 / 3^{\text {rd }}$ of $\mathrm{N}$ were applied before transplanting. Rest of $\mathrm{N}$ was applied in two installments at 15 and 45 days after transplanting. T. Aus rice was harvested on 25 July, 2015 and 26 July, 2016.

For T. Aman rice, the variety Binadhan-7 was transplanted on 29 July 2015 and 30 July 2016 maintaining $20 \times 15 \mathrm{~cm}$ plant spacing. T. Aman rice was harvested on November 5, 2015 and November 7, 2016.

Predetermined plant parameters data were taken as per experimental design.

Statistix 10 was used to determine the significant differences between the treatments. Rice equivalent yield (REY) using formula given by Ahlawal \& Sharma (1993), Where,

Rice equivalent yield ( $\left.\mathrm{t} \mathrm{ha}^{-1} \mathrm{yr}^{-1}\right)=$

Yield of Each Crop ( $\mathrm{tha}^{-1}$ ) x Economic value of Respective crop Tk. ha ${ }^{-1}$

market price of rice

Crop 


\section{Results and Discussion}

Potato

Yield and yield contributing characters of potato as influenced by the fertilizer (Tables 2a and 2b) for two years. Fertilizer treatments significantly influenced plant height, tubers plant ${ }^{-1}$, tuber wt. plant ${ }^{-1}$, tuber yield $\left(\mathrm{t} \mathrm{ha}^{-1}\right)$ of potato. The highest plant height $(66.40$ and $65.87 \mathrm{~cm})$, tubers plant ${ }^{-1}(11.26$ and 11.06), tuber weight plant $^{-1}(0.58 \mathrm{~kg}$ and $0.56 \mathrm{~kg})$ was recorded from the treatment $\mathrm{T}_{6}$ where $25 \%$ extra NPK was added with $100 \%$ STB fertilizer rate in two consecutive years. The lowest plant height $(38.60$ and $39.24 \mathrm{~cm})$, tubers plant ${ }^{-1}$ $\left(6.40\right.$ and 6.12) and tuber weight plant ${ }^{-1}(0.24$ and $0.23 \mathrm{~kg})$ was obtained from control followed by extra 75\% of 100\% STB. The highest tuber yield of potato (25.70 t ha-1 and $24.80 \mathrm{t} \mathrm{ha}^{-1}$ ) was produced from the treatment $\mathrm{T}_{6}$ which out yielded all other treatments. The control treatment produced the lowest tuber yield (10.75 t ha ${ }^{-1}$ and $12.95 \mathrm{t} \mathrm{ha}^{-1}$ ) of potato. Saha et al. (2016) recorded higher tuber yield of potato from $20 \%$ more than the STB dose in potato-maizeT. Aman cropping pattern at AEZ-3. Ali et al. (2009) documented lowest number of tubers plant ${ }^{-1}$, tuber wt. plant ${ }^{-1}$ and tuber yield from control treatment in potato-boro- T. Aman cropping pattern and Mollah et al. (2011) had lowest yield from control treatment in potato-mungbean-T. Aman rice cropping pattern. Chowdhury et al. (2017) reported $64.3 \%$ higher tuber yield of potato in four crop cropping pattern over three crop based cropping pattern.

Table 2a. Plant height, yield and yield contributing characters of potato as influenced by fertilizer management at Jashore during 2014-2015

\begin{tabular}{c|c|c|c|c}
\hline Treatments & $\begin{array}{c}\text { Plant height } \\
(\mathrm{cm})\end{array}$ & $\begin{array}{c}\text { Tubers plant } \\
(\mathrm{no})\end{array}$ & $\begin{array}{c}\text { Tuber wt. plant } \\
(\mathrm{kg})\end{array}$ & $\begin{array}{c}\text { Tuber yield } \\
\left(\mathrm{t} \mathrm{ha}^{-1}\right)\end{array}$ \\
\hline $\mathrm{T}_{1}$ & $62.13 \mathrm{de}$ & $8.00 \mathrm{bc}$ & $0.40 \mathrm{de}$ & $17.89 \mathrm{~cd}$ \\
$\mathrm{~T}_{2}$ & $63.86 \mathrm{~cd}$ & $9.00 \mathrm{bc}$ & $0.48 \mathrm{bc}$ & $21.27 \mathrm{~b}$ \\
$\mathrm{~T}_{3}$ & $66.06 \mathrm{ab}$ & $9.46 \mathrm{~b}$ & $0.50 \mathrm{~b}$ & $22.78 \mathrm{~b}$ \\
$\mathrm{~T}_{4}$ & $64.20 \mathrm{bc}$ & $9.13 \mathrm{bc}$ & $0.48 \mathrm{bc}$ & $21.72 \mathrm{~b}$ \\
$\mathrm{~T}_{5}$ & $63.40 \mathrm{~cd}$ & $8.66 \mathrm{bc}$ & $0.44 \mathrm{~cd}$ & $18.58 \mathrm{c}$ \\
$\mathrm{T}_{6}$ & $66.40 \mathrm{a}$ & $11.26 \mathrm{a}$ & $0.58 \mathrm{a}$ & $25.70 \mathrm{a}$ \\
$\mathrm{T}_{7}$ & $60.93 \mathrm{e}$ & $7.86 \mathrm{~cd}$ & $0.37 \mathrm{e}$ & $16.00 \mathrm{~d}$ \\
$\mathrm{~T}_{8}$ & $38.60 \mathrm{f}$ & $6.40 \mathrm{~d}$ & $0.24 \mathrm{f}$ & $10.75 \mathrm{e}$ \\
\hline $\mathrm{CV}(\%)$ & 6.86 & 9.75 & 6.41 & 5.94 \\
\hline
\end{tabular}

$\mathrm{T}_{1}: 100 \%$ STB $\left(\mathrm{N}_{157} \mathrm{P}_{16} \mathrm{~K}_{35} \mathrm{~S}_{7.2} \mathrm{Zn}_{0.71} \mathrm{~B}_{0.85} \mathrm{Kg} / \mathrm{ha}\right), \mathrm{T}_{2}: \mathrm{T}_{1}+25 \% \mathrm{~N}, \mathrm{~T}_{3}: \mathrm{T}_{1}+25 \% \mathrm{NP}, \mathrm{T}_{4}$ : $\mathrm{T}_{1}+25 \% \mathrm{NK}, \mathrm{T}_{5}: \mathrm{T}_{1}+25 \% \mathrm{PK}, \mathrm{T}_{6}: \mathrm{T}_{1}+25 \% \mathrm{NPK}, \mathrm{T}_{7}: 75 \%$ of $\mathrm{T}_{1}, \mathrm{~T}_{8}:$ native fertility (control).

Table 2b. Plant height yield and yield contributing characters of potato as influenced by fertilizer management at Jashore during 2015-2016

\begin{tabular}{c|c|c|c|c}
\hline Treatments & $\begin{array}{c}\text { Plant height } \\
(\mathrm{cm})\end{array}$ & $\begin{array}{c}\text { Tubers plant } \\
(\mathrm{no})\end{array}$ & $\begin{array}{c}\text { Tuber wt. plant } \\
(\mathrm{kg})\end{array}$ & $\begin{array}{c}\text { Tuber yield } \\
\left(\mathrm{t} \mathrm{ha} \mathrm{H}^{-1}\right)\end{array}$ \\
\hline $\mathrm{T}_{1}$ & $61.92 \mathrm{de}$ & $8.30 \mathrm{bc}$ & $0.38 \mathrm{de}$ & $16.97 \mathrm{~cd}$ \\
$\mathrm{~T}_{2}$ & $62.35 \mathrm{~cd}$ & $9.10 \mathrm{bc}$ & $0.45 \mathrm{bc}$ & $20.92 \mathrm{~b}$ \\
$\mathrm{~T}_{3}$ & $65.12 \mathrm{ab}$ & $9.54 \mathrm{~b}$ & $0.47 \mathrm{~b}$ & $21.89 \mathrm{~b}$ \\
$\mathrm{~T}_{4}$ & $63.33 \mathrm{bc}$ & $9.19 \mathrm{bc}$ & $0.45 \mathrm{bc}$ & $20.84 \mathrm{~b}$ \\
$\mathrm{~T}_{5}$ & $62.54 \mathrm{~cd}$ & $8.42 \mathrm{bc}$ & $0.40 \mathrm{~cd}$ & $17.86 \mathrm{c}$ \\
$\mathrm{T}_{6}$ & $65.87 \mathrm{a}$ & $11.06 \mathrm{a}$ & $0.56 \mathrm{a}$ & $24.80 \mathrm{a}$ \\
$\mathrm{T}_{7}$ & $59.83 \mathrm{e}$ & $7.14 \mathrm{~cd}$ & $0.35 \mathrm{e}$ & $15.92 \mathrm{~d}$ \\
$\mathrm{~T}_{8}$ & $39.24 \mathrm{f}$ & $6.12 \mathrm{~d}$ & $0.23 \mathrm{f}$ & $12.95 \mathrm{e}$ \\
\hline $\mathrm{CV}(\%)$ & 7.79 & 9.63 & 5.98 & 6.22
\end{tabular}

$\mathrm{T}_{1}: 100 \% \mathrm{STB}\left(\mathrm{N}_{157} \mathrm{P}_{16} \mathrm{~K}_{35} \mathrm{~S}_{7.2} \mathrm{Zn}_{0.71} \mathrm{~B}_{0.85} \mathrm{Kg} / \mathrm{ha}\right), \mathrm{T}_{2}: \mathrm{T}_{1}+25 \% \mathrm{~N}, \mathrm{~T}_{3}: \mathrm{T}_{1}+25 \% \mathrm{NP}, \mathrm{T}_{4}$ : $\mathrm{T}_{1}+25 \% \mathrm{NK}, \mathrm{T}_{5}: \mathrm{T}_{1}+25 \% \mathrm{PK}, \mathrm{T}_{6}: \mathrm{T}_{1}+25 \% \mathrm{NPK}, \mathrm{T}_{7}: 75 \%$ of $\mathrm{T}_{1}, \mathrm{~T}_{8}:$ native fertility (control). 


\section{Boro}

The second component of the cropping pattern was Boro rice. Plant height, yield and yield components of Boro rice have been presented in Tables 3a and 3b. Most of the studied parameters of Boro rice were significantly influenced by fertilizer treatments. In 2015, the maximum plant height $(96.21 \mathrm{~cm})$ was obtained from $T_{5}$ treatment which is statistically at par with $T_{2}, T_{3}, T_{4}$ and $T_{6}$. In 2016, the maximum plant $\left(96.02 \mathrm{~cm}\right.$ ) was recorded from $\mathrm{T}_{6}$ treatment which is statistically at par with $T_{2}, T_{3}, T_{4}$ and $T_{5}$. Tillers hill ${ }^{-1}$, panicles hill ${ }^{-1}$, panicle length and straw yields were significantly higher over control treatment.

Table 3a. Plant height, yield and yield contributing characters of Boro rice as influenced by fertilizer management during 2015

\begin{tabular}{|c|c|c|c|c|c|c|c|}
\hline \multirow{2}{*}{ Treatments } & \multirow{2}{*}{$\begin{array}{l}\text { Plant } \\
\text { height } \\
(\mathrm{cm})\end{array}$} & $\begin{array}{c}\text { No. of } \\
\text { tiller }\end{array}$ & $\begin{array}{l}\text { No. of } \\
\text { panicle }\end{array}$ & \multirow{2}{*}{$\begin{array}{l}\text { Panicle } \\
\text { length } \\
\text { (cm) }\end{array}$} & \multirow{2}{*}{$\begin{array}{l}\text { 1000- } \\
\text { grain } \\
\text { wt.(g) }\end{array}$} & $\begin{array}{l}\text { Straw } \\
\text { yield }\end{array}$ & $\begin{array}{l}\text { Grain } \\
\text { yield }\end{array}$ \\
\hline & & \multicolumn{2}{|c|}{ hill $^{-1}$} & & & \multicolumn{2}{|c|}{$\left(\mathrm{t} \mathrm{ha}^{-1}\right)$} \\
\hline $\mathrm{T}_{1}$ & $87.12 \mathrm{c}$ & $19.92 \mathrm{a}$ & $19.23 a$ & $19.60 \mathrm{a}$ & 22.50 & $6.35 a$ & $5.50 \mathrm{ab}$ \\
\hline $\mathrm{T}_{2}$ & $91.01 \mathrm{ab}$ & $19.81 \mathrm{a}$ & $19.01 \mathrm{a}$ & $19.61 \mathrm{a}$ & 23.31 & $6.23 a$ & $5.61 \mathrm{ab}$ \\
\hline $\mathrm{T}_{3}$ & $92.10 \mathrm{ab}$ & $20.02 a$ & $19.12 \mathrm{a}$ & $19.91 \mathrm{a}$ & 22.03 & $6.24 a$ & $6.26 \mathrm{a}$ \\
\hline $\mathrm{T}_{4}$ & $95.11 \mathrm{a}$ & $19.91 \mathrm{a}$ & $19.21 \mathrm{a}$ & $19.92 \mathrm{a}$ & 21.50 & $6.43 a$ & $5.82 \mathrm{ab}$ \\
\hline $\mathrm{T}_{5}$ & $96.21 \mathrm{a}$ & $20.22 \mathrm{a}$ & $19.32 \mathrm{a}$ & $19.60 \mathrm{a}$ & 21.90 & $6.12 \mathrm{a}$ & $5.80 \mathrm{ab}$ \\
\hline $\mathrm{T}_{6}$ & $94.24 \mathrm{a}$ & $20.12 \mathrm{a}$ & $19.62 \mathrm{a}$ & $19.81 \mathrm{a}$ & 23.31 & $6.15 a$ & $6.50 \mathrm{a}$ \\
\hline $\mathrm{T}_{7}$ & $88.04 b c$ & $20.02 \mathrm{a}$ & $19.22 \mathrm{a}$ & $19.31 \mathrm{a}$ & 21.21 & $5.78 \mathrm{a}$ & $5.24 a b$ \\
\hline $\mathrm{T}_{8}$ & $78.18 \mathrm{~d}$ & $15.11 \mathrm{~b}$ & $14.42 \mathrm{~b}$ & $16.50 \mathrm{~b}$ & 20.91 & $4.34 \mathrm{~b}$ & $4.36 \mathrm{~b}$ \\
\hline $\mathrm{CV}(\%)$ & 3.68 & 2.98 & 2.86 & 2.11 & 4.25 & 9.94 & 10.43 \\
\hline
\end{tabular}

$\mathrm{T}_{1}: 100 \%$ STB $\left(\mathrm{N}_{186} \mathrm{P}_{30} \mathrm{~K}_{23} \mathrm{~S}_{30} \mathrm{Kg} / \mathrm{ha}\right), \mathrm{T}_{2}: \mathrm{T}_{1}+25 \% \mathrm{~N}, \mathrm{~T}_{3}: \mathrm{T}_{1}+25 \% \mathrm{NP}, \mathrm{T}_{4}: \mathrm{T}_{1}+25 \%$ NK, $T_{5}: T_{1}+25 \%$ PK, $T_{6}: T_{1}+25 \%$ NPK, $T_{7}: 75 \%$ of $\mathrm{T}_{1}, \mathrm{~T}_{8}$ : control or native fertility.

Table 3b. Plant height, yield and yield contributing characters of Boro rice as influenced by fertilizer management during 2016

\begin{tabular}{|c|c|c|c|c|c|c|c|}
\hline \multirow{2}{*}{ Treatments } & \multirow{2}{*}{$\begin{array}{l}\text { Plant } \\
\text { height } \\
\text { (cm) }\end{array}$} & $\begin{array}{c}\text { No. of } \\
\text { tiller }\end{array}$ & $\begin{array}{l}\text { No. of } \\
\text { panicle }\end{array}$ & \multirow{2}{*}{$\begin{array}{l}\text { Panicle } \\
\text { length } \\
\text { (cm) }\end{array}$} & \multirow{2}{*}{$\begin{array}{l}1000- \\
\text { grain } \\
\text { wt.(g) }\end{array}$} & $\begin{array}{c}\text { Straw } \\
\text { yield }\end{array}$ & Grain yield \\
\hline & & & & & & \multicolumn{2}{|c|}{$\left(\mathrm{t} \mathrm{ha}^{-1}\right)$} \\
\hline $\mathrm{T}_{1}$ & $86.04 c$ & $19.85 a$ & $19.15 a$ & $19.50 \mathrm{a}$ & 22.41 & $6.20 a$ & $5.40 \mathrm{ab}$ \\
\hline $\mathrm{T}_{2}$ & 89.78ab & $19.73 a$ & $19.02 a$ & $19.51 \mathrm{a}$ & 23.23 & $6.13 a$ & $5.56 \mathrm{ab}$ \\
\hline $\mathrm{T}_{3}$ & 90.98ab & $20.00 \mathrm{a}$ & $19.06 \mathrm{a}$ & $19.80 \mathrm{a}$ & 22.02 & $6.22 \mathrm{a}$ & $6.31 \mathrm{a}$ \\
\hline $\mathrm{T}_{4}$ & $94.02 \mathrm{a}$ & $19.84 a$ & $19.10 \mathrm{a}$ & $19.83 a$ & 21.40 & $6.40 a$ & $5.72 \mathrm{ab}$ \\
\hline $\mathrm{T}_{5}$ & $95.05 a$ & $20.18 a$ & $19.23 a$ & $19.55 \mathrm{a}$ & 21.80 & $6.01 \mathrm{a}$ & $5.70 \mathrm{ab}$ \\
\hline $\mathrm{T}_{6}$ & $96.02 \mathrm{a}$ & $20.13 a$ & $19.52 a$ & $19.70 \mathrm{a}$ & 23.24 & $6.03 a$ & $6.31 \mathrm{a}$ \\
\hline $\mathrm{T}_{7}$ & $87.08 \mathrm{bc}$ & $20.02 \mathrm{a}$ & $19.11 \mathrm{a}$ & $19.24 a$ & 21.13 & $5.71 \mathrm{a}$ & $5.15 \mathrm{ab}$ \\
\hline $\mathrm{T}_{8}$ & $77.06 \mathrm{~d}$ & $15.04 \mathrm{~b}$ & $14.55 \mathrm{~b}$ & $16.41 \mathrm{~b}$ & 20.80 & $4.36 \mathrm{~b}$ & $4.32 \mathrm{~b}$ \\
\hline CV (\%) & 5.34 & 3.48 & 3.12 & 2.12 & 5.18 & 9.72 & 9.16 \\
\hline
\end{tabular}

$\mathrm{T}_{1}: 100 \% \operatorname{STB}\left(\mathrm{N}_{186} \mathrm{P}_{30} \mathrm{~K}_{23} \mathrm{~S}_{30} \mathrm{Kg} / \mathrm{ha}\right), \mathrm{T}_{2}: \mathrm{T}_{1}+25 \% \mathrm{~N}, \mathrm{~T}_{3}: \mathrm{T}_{1}+25 \% \mathrm{NP}, \mathrm{T}_{4}: \mathrm{T}_{1}+25 \%$ NK, $\mathrm{T}_{5}: \mathrm{T}_{1}+25 \% \mathrm{PK}, \mathrm{T}_{6}: \mathrm{T}_{1}+25 \% \mathrm{NPK}, \mathrm{T}_{7}: 75 \%$ of $\mathrm{T}_{1}, \mathrm{~T}_{8}$ : control or native fertility.

\section{T. Aus}

T. Aus rice was transplanted as the third crop of the pattern. The variety used for $\mathrm{T}$. Aus rice was BRRI dhan48, a short duration variety. Most of the yield 
contributing characters and grain yield was significantly influenced by fertilizer treatments. The maximum plant height $(94.55$ and $93.53 \mathrm{~cm})$ was recorded from the treatment $T_{5}$ which was statistically identical to $T_{4}, T_{6}, T_{2}$ and $T_{3}$. The lowest plant height $(76.54$ and $75.53 \mathrm{~cm})$ was obtained from the control. There is no significant variation among the treatments except native fertility treatment in case of number of tillers hill ${ }^{-1}$, number of panicle hill ${ }^{-1}$, panicle length and straw yield. The highest grain yield of $\mathrm{T}$. Aus rice (3.13 t ha ${ }^{-1}$ and $\left.6.10 \mathrm{t} \mathrm{ha}^{-1}\right)$ was produced by the treatment $\mathrm{T}_{6}$ where $25 \%$ additional NPK was added with the $100 \%$ STB rates (Tables 4a and 4b). The control treatment produced the lowest rice yield. The native fertility treatment produced the lowest grain yield $(1.92 \mathrm{t}$ $\mathrm{ha}^{-1}$ and $\left.1.84 \mathrm{t} \mathrm{ha}^{-1}\right)$ of $\mathrm{T}$. Aus rice. The maximum grain yield $\left(6.26 \mathrm{t} \mathrm{ha}^{-1}\right.$ in 2015 and $6.31 \mathrm{t} \mathrm{ha}^{-1}$ in 2016) was obtained from $\mathrm{T}_{6}$ which followed by other fertilizer variables but significantly different from control $\left(4.36 \mathrm{t} \mathrm{ha}^{-1}\right.$ and $4.32 \mathrm{t}$ $\left.\mathrm{ha}^{-1}\right)$. This result was in agreement with Hasan et al. (2017) who opined that an increase of $\mathrm{N}, \mathrm{P}$ and $\mathrm{K}$ fertilizer doses from STB recommended doses significantly increased yield and yield contributing parameters of rice (Chaudhary et al., 2011); Yoseftabar, 2012).

Table 4a. Plant height, yield and yield contributing characters of T. Aus rice during 2015

\begin{tabular}{|c|c|c|c|c|c|c|c|}
\hline \multirow{2}{*}{ Treatments } & \multirow{2}{*}{ Plant height $(\mathrm{cm})$} & No. of tiller & No. of panicle & \multirow{2}{*}{$\begin{array}{l}\text { Panicle } \\
\text { length } \\
\text { (cm) }\end{array}$} & \multirow{2}{*}{$\begin{array}{l}1000- \\
\text { grain } \\
\text { wt.(g) }\end{array}$} & $\begin{array}{l}\text { Straw } \\
\text { yield }\end{array}$ & $\begin{array}{l}\text { Grain } \\
\text { yield }\end{array}$ \\
\hline & & \multicolumn{2}{|c|}{ hill $^{-1}$} & & & \multicolumn{2}{|c|}{$\left(\mathrm{t} \mathrm{ha}^{-1}\right)$} \\
\hline $\mathrm{T}_{1}$ & $81.26 c$ & $16.71 \mathrm{a}$ & $16.21 \mathrm{a}$ & $16.62 a$ & 19.51 & $4.42 \mathrm{a}$ & $2.83 \mathrm{ab}$ \\
\hline $\mathrm{T}_{2}$ & $90.58 \mathrm{ab}$ & $16.72 \mathrm{a}$ & $16.02 \mathrm{a}$ & $16.63 a$ & 19.33 & $4.21 \mathrm{a}$ & $2.82 \mathrm{ab}$ \\
\hline $\mathrm{T}_{3}$ & $90.35 \mathrm{ab}$ & $17.01 \mathrm{a}$ & $16.11 \mathrm{a}$ & $16.92 \mathrm{a}$ & 19.01 & $4.01 \mathrm{a}$ & $2.82 \mathrm{ab}$ \\
\hline $\mathrm{T}_{4}$ & $93.45 a$ & $16.72 \mathrm{a}$ & $16.22 \mathrm{a}$ & $16.91 \mathrm{a}$ & 19.52 & $4.62 \mathrm{a}$ & $2.83 \mathrm{ab}$ \\
\hline $\mathrm{T}_{5}$ & $94.55 a$ & $17.23 \mathrm{a}$ & $16.31 \mathrm{a}$ & $16.62 a$ & 19.92 & $4.01 \mathrm{a}$ & $2.92 \mathrm{ab}$ \\
\hline $\mathrm{T}_{6}$ & $92.98 \mathrm{a}$ & $17.11 \mathrm{a}$ & $16.62 \mathrm{a}$ & $16.81 \mathrm{a}$ & 19.31 & $4.02 \mathrm{a}$ & 3.13 a \\
\hline $\mathrm{T}_{7}$ & $86.32 b c$ & $17.02 \mathrm{a}$ & $16.21 \mathrm{a}$ & $16.32 \mathrm{a}$ & 19.43 & $3.81 \mathrm{a}$ & $2.11 b$ \\
\hline $\mathrm{T}_{8}$ & $76.54 \mathrm{~d}$ & $15.13 b$ & $14.42 \mathrm{~b}$ & $14.51 b$ & 19.92 & $2.41 \mathrm{~b}$ & $1.92 \mathrm{~b}$ \\
\hline CV (\%) & 3.56 & 3.56 & 4.12 & 2.27 & 4.13 & 9.68 & 9.45 \\
\hline
\end{tabular}

$\mathrm{T}_{1}: 100 \% \mathrm{STB}\left(\mathrm{N}_{190} \mathrm{P}_{15} \mathrm{~K}_{2.17} \mathrm{~S}_{23} \mathrm{Kg} / \mathrm{ha}\right), \mathrm{T}_{2}: \mathrm{T}_{1}+25 \% \mathrm{~N}, \mathrm{~T}_{3}: \mathrm{T}_{1}+25 \% \mathrm{NP}, \mathrm{T}_{4}: \mathrm{T}_{1}+25 \%$

$\mathrm{NK}, \mathrm{T}_{5}: \mathrm{T}_{1}+25 \% \mathrm{PK}, \mathrm{T}_{6}: \mathrm{T}_{1}+25 \% \mathrm{NPK}, \mathrm{T}_{7}: 75 \%$ of $\mathrm{T}_{1}, \mathrm{~T}_{8}$ : control or native fertility.

Table 4b. Plant height, yield and yield contributing characters of $\mathrm{T}$. Aus rice during 2016

\begin{tabular}{|c|c|c|c|c|c|c|c|}
\hline \multirow{2}{*}{ Treatments } & \multirow{2}{*}{ Plant height $(\mathrm{cm})$} & No. of tiller & No. of panicle & \multirow{2}{*}{$\begin{array}{l}\text { Panicle } \\
\text { length } \\
(\mathrm{cm})\end{array}$} & \multirow{2}{*}{$\begin{array}{c}1000- \\
\text { grain } \\
\text { wt.(g) }\end{array}$} & $\begin{array}{c}\text { Straw } \\
\text { yield }\end{array}$ & $\begin{array}{l}\text { Grain } \\
\text { yield }\end{array}$ \\
\hline & & \multicolumn{2}{|c|}{ hill $^{-1}$} & & & \multicolumn{2}{|c|}{$(\mathrm{t} \mathrm{ha-1})$} \\
\hline $\mathrm{T}_{1}$ & $81.22 \mathrm{c}$ & $15.51 a$ & $15.11 \mathrm{a}$ & $15.63 a$ & 19.45 & $4.33 a$ & $2.74 a b$ \\
\hline $\mathrm{T}_{2}$ & $89.34 a b$ & $15.62 \mathrm{a}$ & $15.12 \mathrm{a}$ & $15.60 \mathrm{a}$ & 19.34 & $4.13 a$ & $2.73 \mathrm{ab}$ \\
\hline $\mathrm{T}_{3}$ & $89.30 \mathrm{ab}$ & $16.11 a$ & $15.02 \mathrm{a}$ & $15.92 \mathrm{a}$ & 19.25 & $4.04 \mathrm{a}$ & $2.72 \mathrm{ab}$ \\
\hline $\mathrm{T}_{4}$ & $92.44 a$ & $15.81 \mathrm{a}$ & $15.14 a$ & $15.91 \mathrm{a}$ & 19.44 & $4.44 a$ & $2.74 a b$ \\
\hline $\mathrm{T}_{5}$ & $93.53 a$ & $16.31 \mathrm{a}$ & $15.23 a$ & $15.62 \mathrm{a}$ & 19.55 & $4.03 a$ & $2.83 \mathrm{ab}$ \\
\hline $\mathrm{T}_{6}$ & $91.92 \mathrm{a}$ & $16.23 a$ & $15.41 \mathrm{a}$ & $15.81 a$ & 19.34 & $4.02 \mathrm{a}$ & $3.10 \mathrm{a}$ \\
\hline $\mathrm{T}_{7}$ & $85.34 b c$ & $16.01 \mathrm{a}$ & $15.15 a$ & $15.32 \mathrm{a}$ & 19.34 & $3.74 a$ & $2.22 \mathrm{~b}$ \\
\hline $\mathrm{T}_{8}$ & $75.53 \mathrm{~d}$ & $14.02 \mathrm{~b}$ & $13.81 \mathrm{~b}$ & $13.51 \mathrm{~b}$ & 19.35 & $2.43 b$ & $1.84 \mathrm{~b}$ \\
\hline $\mathrm{CV}(\%)$ & 4.15 & 3.27 & 3.15 & 3.14 & 4.97 & 9.32 & 9.23 \\
\hline
\end{tabular}

$\mathrm{T}_{1}: 100 \%$ STB $\left(\mathrm{N}_{190} \mathrm{P}_{15} \mathrm{~K}_{2.17} \mathrm{~S}_{23} \mathrm{Kg} / \mathrm{ha}\right), \mathrm{T}_{2}: \mathrm{T}_{1}+25 \% \mathrm{~N}, \mathrm{~T}_{3}: \mathrm{T}_{1}+25 \% \mathrm{NP}, \mathrm{T}_{4}: \mathrm{T}_{1}+25 \%$

$\mathrm{NK}, \mathrm{T}_{5}: \mathrm{T}_{1}+25 \% \mathrm{PK}, \mathrm{T}_{6}: \mathrm{T}_{1}+25 \% \mathrm{NPK}, \mathrm{T}_{7}: 75 \%$ of $\mathrm{T}_{1}, \mathrm{~T}_{8}$ : control or native fertility. 


\section{T. Aman}

The fourth component of the cropping pattern was $\mathrm{T}$. Aman rice. The yield contributing characters and grain yield of $\mathrm{T}$. Aman rice are presented in Tables $5 \mathrm{a}$ and $5 \mathrm{~b}$. Most of the yield contributing characters and straw and grain yield of T. Aman rice was significantly influenced by fertilizer treatments. High plant height $\left(97.68\right.$ and $97.58 \mathrm{~cm}$ ) was recorded from the treatment $\mathrm{T}_{6}$ where $25 \%$ extra NPK was added over the 100\% STB fertilizer rate which was statistically identical with $T_{5}$ followed by $T_{1}, T_{2}, T_{3}$ and $T_{4}$. The lowest plant height $(71.58$ and $71.48 \mathrm{~cm}$ ) was obtained from the control. The maximum number of tiller hill $^{-1}$ (17.32 and 16.16) was obtained from $\mathrm{T}_{6}$ treatment which was statistically identical with rest of the treatment except $\mathrm{T}_{8}$ which produced lowest number of tillers hill $^{-1}$ (9.92 and 9.66) in two years). There is no significant variation among the treatments in case of panicle length and 1000-grain weight. The highest grain yield (3.95 $\mathrm{t} \mathrm{ha}{ }^{-1}$ and $3.98 \mathrm{t} \mathrm{ha}^{-1}$ ) was obtained from the treatment $\mathrm{T}_{6}$ where 25\% extra NPK was applied where control treatment produced the lowest grain yield (2.12 $\mathrm{t} \mathrm{ha}^{-1}$ and $\left.2.17 \mathrm{t} \mathrm{ha}^{-1}\right)$. Ali et al. (2009) reported the highest grain yield from soil test value for $\mathrm{HYG}$ and lowest from control treatment. Saha et al. (2016) also documented higher yield from 20\% more than the STB dose in potato-maize-T. Aman cropping pattern of AEZ-3.

Table 5a. Plant height, yield and yield contributing characters of T. Aman rice in 2015

\begin{tabular}{|c|c|c|c|c|c|c|c|}
\hline \multirow{2}{*}{ Treatments } & \multirow{2}{*}{ Plant height $(\mathrm{cm})$} & No. of tiller & No. of panicle & \multirow{2}{*}{$\begin{array}{l}\text { Panicle } \\
\text { length } \\
\text { (cm) }\end{array}$} & \multirow{2}{*}{$\begin{array}{l}\text { 1000- } \\
\text { grain } \\
\text { wt.(g) }\end{array}$} & $\begin{array}{c}\text { Straw } \\
\text { yield }\end{array}$ & $\begin{array}{l}\text { Grain } \\
\text { yield }\end{array}$ \\
\hline & & \multicolumn{2}{|c|}{ hill $^{-1}$} & & & \multicolumn{2}{|c|}{$\left(\mathrm{t} \mathrm{ha}^{-1}\right)$} \\
\hline $\mathrm{T}_{1}$ & 95.38ab & $15.91 \mathrm{a}$ & $15.22 \mathrm{~b}$ & 18.22 & 21.02 & $5.61 \mathrm{ab}$ & $3.51 \mathrm{a}$ \\
\hline $\mathrm{T}_{2}$ & 95.90ab & $17.02 \mathrm{a}$ & $16.31 \mathrm{ab}$ & 18.31 & 21.61 & 5.12abc & $3.53 a$ \\
\hline $\mathrm{T}_{3}$ & $96.42 \mathrm{ab}$ & $17.12 \mathrm{a}$ & $16.52 \mathrm{ab}$ & 18.12 & 21.01 & $5.32 \mathrm{abc}$ & $3.62 \mathrm{a}$ \\
\hline $\mathrm{T}_{4}$ & 96.59ab & $16.91 \mathrm{a}$ & 16.31ab & 18.21 & 21.82 & $4.83 b c$ & $3.52 \mathrm{a}$ \\
\hline $\mathrm{T}_{5}$ & $97.66 a$ & $17.12 \mathrm{a}$ & $16.42 \mathrm{ab}$ & 18.42 & 21.91 & $5.83 \mathrm{ab}$ & $3.44 a$ \\
\hline 15 & & & & 18.21 & 21.12 & & $3.95 a$ \\
\hline $\mathrm{T}_{6}$ & $97.68 a$ & $17.32 \mathrm{a}$ & $16.82 \mathrm{a}$ & & & $\begin{array}{l}5.92 \mathrm{a} \\
4.32 \mathrm{bc}\end{array}$ & \\
\hline $\mathrm{T}_{7}$ & $93.76 b$ & $16.01 \mathrm{a}$ & 15.41ab & 18.31 & 21.22 & & $3.21 a$ \\
\hline $\mathrm{T}_{8}$ & $71.58 \mathrm{c}$ & $9.92 \mathrm{~b}$ & $9.22 \mathrm{c}$ & 18.02 & 21.32 & $3.91 \mathrm{c}$ & $2.12 \mathrm{~b}$ \\
\hline $\mathrm{CV}(\%)$ & 3.14 & 4.86 & 4.76 & 4.97 & 3.76 & 10.80 & 3.98 \\
\hline
\end{tabular}

$\mathrm{T}_{1}: 100 \%$ STB $\left(\mathrm{N}_{159} \mathrm{P}_{30} \mathrm{~K}_{7} \mathrm{~S}_{24} \mathrm{Kg} / \mathrm{ha}\right), \mathrm{T}_{2}: \mathrm{T}_{1}+25 \% \mathrm{~N}, \mathrm{~T}_{3}: \mathrm{T}_{1}+25 \% \mathrm{NP}, \mathrm{T}_{4}: \mathrm{T}_{1}+25 \%$ NK, $\mathrm{T}_{5}: \mathrm{T}_{1}+25 \% \mathrm{PK}, \mathrm{T}_{6}: \mathrm{T}_{1}+25 \% \mathrm{NPK}, \mathrm{T}_{7}: 75 \%$ of $\mathrm{T}_{1}, \mathrm{~T}_{8}$ : control or native fertility.

Table 5b. Plant height, yield and yield contributing characters of $\mathrm{T}$. Aman rice in 2016

\begin{tabular}{|c|c|c|c|c|c|c|c|}
\hline \multirow{2}{*}{ Treatments } & \multirow{2}{*}{$\begin{array}{l}\text { Plant height } \\
\text { (cm) }\end{array}$} & $\begin{array}{c}\text { No. of } \\
\text { tiller }\end{array}$ & No. of panicle & \multirow{2}{*}{$\begin{array}{l}\text { Panicle } \\
\text { length } \\
\text { (cm) }\end{array}$} & \multirow{2}{*}{$\begin{array}{l}1000 \\
\text { grain } \\
\text { wt.(g) }\end{array}$} & $\begin{array}{l}\text { Straw } \\
\text { yield }\end{array}$ & $\begin{array}{l}\text { Grain } \\
\text { yield }\end{array}$ \\
\hline & & \multicolumn{2}{|c|}{ hill $^{-1}$} & & & \multicolumn{2}{|c|}{$\left(\mathrm{t} \mathrm{ha}^{-1}\right)$} \\
\hline $\mathrm{T}_{1}$ & $95.50 \mathrm{ab}$ & $15.81 \mathrm{a}$ & $15.17 b$ & 18.40 & 21.05 & $5.17 \mathrm{ab}$ & $3.58 \mathrm{a}$ \\
\hline $\mathrm{T}_{2}$ & 95.53ab & $16.82 \mathrm{a}$ & $16.28 \mathrm{ab}$ & 18.01 & 21.54 & $5.08 a b c$ & $3.58 \mathrm{a}$ \\
\hline $\mathrm{T}_{3}$ & $96.52 \mathrm{ab}$ & $16.73 a$ & $16.45 \mathrm{ab}$ & 18.90 & 21.26 & $5.08 \mathrm{abc}$ & $3.65 \mathrm{a}$ \\
\hline $\mathrm{T}_{4}$ & $96.41 \mathrm{ab}$ & $15.94 a$ & $15.58 \mathrm{ab}$ & 18.60 & 21.14 & $4.87 \mathrm{bc}$ & $3.47 a$ \\
\hline $\mathrm{T}_{5}$ & $97.56 a$ & $16.01 a$ & $15.86 \mathrm{ab}$ & 18.32 & 21.56 & $5.78 \mathrm{ab}$ & $3.55 \mathrm{a}$ \\
\hline $\mathrm{T}_{6}$ & $97.58 \mathrm{a}$ & $16.16 a$ & $16.15 \mathrm{a}$ & 18.11 & 21.55 & 587 ב & $3.98 \mathrm{a}$ \\
\hline $\mathrm{T}_{7}$ & $93.63 b$ & $15.73 a$ & $15.17 \mathrm{ab}$ & 18.72 & 21.34 & $4.58 \mathrm{bc}$ & $3.28 \mathrm{a}$ \\
\hline $\mathrm{T}_{8}$ & $71.48 c$ & $9.66 \mathrm{~b}$ & $9.03 c$ & 18.51 & 21.26 & $3.87 \mathrm{c}$ & $2.17 \mathrm{~b}$ \\
\hline CV (\%) & 3.78 & 5.34 & 4.98 & 5.92 & 3.56 & 9.87 & 7.58 \\
\hline
\end{tabular}


$\mathrm{T}_{1}: 100 \% \operatorname{STB}\left(\mathrm{N}_{159} \mathrm{P}_{30} \mathrm{~K}_{7} \mathrm{~S}_{24} \mathrm{Kg} / \mathrm{ha}\right), \mathrm{T}_{2}: \mathrm{T}_{1}+25 \% \mathrm{~N}, \mathrm{~T}_{3}: \mathrm{T}_{1}+25 \% \mathrm{NP}, \mathrm{T}_{4}: \mathrm{T}_{1}+25 \%$ $\mathrm{NK}, \mathrm{T}_{5}: \mathrm{T}_{1}+25 \% \mathrm{PK}, \mathrm{T}_{6}: \mathrm{T}_{1}+25 \% \mathrm{NPK}, \mathrm{T}_{7}: 75 \%$ of $\mathrm{T}_{1}, \mathrm{~T}_{8}$ : control or native fertility.

Rice equivalent yield

To compare among the treatments, potato yield was converted into rice equivalent yield on the basis of prevailing market price of individual crop. The highest rice equivalent yield was recorded from $\mathrm{T}_{6}$ treatment due to the highest yield of potato, boro rice, $T$. Aus rice and $T$. Aman rice. Treatment $T_{8}$ showed lower rice equivalent yield due to lower yield of all the four crops (Table 6). Ali et al. (2009) also documented significant difference in rice system yield due to different fertilizer doses.

Table 6. Rice equivalent yield of Potato-Boro-T. Aus-T. Aman cropping pattern

\begin{tabular}{c|c|c|c|c|c}
\hline \multirow{2}{*}{ Treatments } & \multicolumn{4}{|c|}{$\begin{array}{c}\text { Yield of crops in the pattern } \\
(\mathrm{t} \mathrm{ha})\end{array}$} & $\begin{array}{c}\text { Rice equivalent } \\
\left.\text { yield (t ha }{ }^{-1}\right)\end{array}$ \\
\cline { 2 - 6 } & Potato & Boro & T. Aus & T. Aman & Potato \\
\hline $\mathrm{T}_{1}$ & 17.43 & 5.45 & 2.79 & 3.55 & 8.72 \\
$\mathrm{~T}_{2}$ & 21.10 & 5.59 & 2.78 & 3.56 & 10.55 \\
$\mathrm{~T}_{3}$ & 22.34 & 6.41 & 2.77 & 3.64 & 11.17 \\
$\mathrm{~T}_{4}$ & 21.28 & 5.77 & 2.79 & 3.50 & 10.64 \\
$\mathrm{~T}_{5}$ & 18.22 & 5.75 & 2.88 & 3.49 & 9.11 \\
$\mathrm{~T}_{6}$ & 25.25 & 6.29 & 3.12 & 3.97 & 12.63 \\
$\mathrm{~T}_{7}$ & 15.96 & 5.20 & 2.17 & 3.25 & 7.98 \\
$\mathrm{~T}_{8}$ & 11.85 & 4.34 & 1.88 & 2.15 & 5.93 \\
\hline
\end{tabular}

Price: $1 \mathrm{~kg}$ potato $=\mathrm{Tk} .10,1 \mathrm{~kg}$ rice $=\mathrm{Tk} .20$

$\mathrm{T}_{1}: 100 \%$ STB, $\mathrm{T}_{2}: \mathrm{T}_{1}+25 \% \mathrm{~N}, \mathrm{~T}_{3}: \mathrm{T}_{1}+25 \% \mathrm{NP}, \mathrm{T}_{4}: \mathrm{T}_{1}+25 \% \mathrm{NK}, \mathrm{T}_{5}: \mathrm{T}_{1}+25 \% \mathrm{PK}$,

$\mathrm{T}_{6}: \mathrm{T}_{1}+25 \% \mathrm{NPK}, \mathrm{T}_{7}: 75 \%$ of $\mathrm{T}_{1}, \mathrm{~T}_{8}$ : control treatment or native fertility.

\section{Conclusion}

From consecutive two years study of four cropping pattern, it could be concluded that STB $+25 \%$ extra NPK performed better on the whole pattern basis than all other fertilizer levels and this fertilizer package could be used for PotatoBoro-T. Aus-T. Aman cropping pattern under Jashore region (AEZ-11).

\section{References}

Ahalwat, I.P.S. and Sharma, R.P. 1993. Agron mid Terminology. $3^{\text {rd }}$ edition, New Delhi, Indian Soc. Agron.

Ali, M.R., D.J. Costa, M.A. Sayed, M.A.H. Khanand and J.A. Abedin. 2009. Development of fertilizer recommendation for the cropping pattern potatoboro-t. aman in irrigated medium highland condition under AEZ -9. Bangladesh J. Agril. Res. 34(1): 41-49.

BBS (Bangladesh Bureau of Statistics). 2012. Statistical Yearbook of Bangladesh. Bangladesh Bureau of Statistics, Ministry of Planning, Dhaka. Bangladesh.

Chaudhary, S.K., J.P. Singh and S. Jhair. 2011. Effect of integrated nitrogen management on yield, quality and nutrient uptake of rice (Oryza sativa L.) under different dates of planting. Indian J. Agron. 56(3): 228-231.

Chowdhury, A.K., T. Zahan, M.M. Anowar, M.K. Islam, S.H. Molla and A.S.M.M.R. Khan. 2017. Improvement of Potato- Jute- T. Aman cropping pattern through inclusion of mungbean. Bangladesh Agron. J. 20(2): 87-95. 
Hasan, A.B.M.M., S.M. Shamsuzzaman, H.A.K. Chowdhury, S.M. Rasel, N.H. Khan, M.E. Rahman, M. Mehnaz and A.W. Samsuri. 2017. Effect of different fertilizer packages on the yield and yield attributes on BRRI dhan47 in saline areas. Bangladesh J. Bot. 46(1): 467-472.

Mollah, M.R.A., N. Islam and M.A.R. Sarkar. 2011. Integrated nutrient management for potato-mungbean-T. aman rice cropping pattern under level barind agro ecological zone. Bangladesh J. Agril. Res. 36(4): 711722.

ORC. 2013. Oilseed Research Centre Annual Research Report. Oilseed Research Centre. Bangladesh Agricultural Research Institute, Joydebpur, Gazipur.

Saha, P.K., F. Rahman, M. Akter, R. Islam, A.T.M.S. Hossain and M.G. Ali. 2016. Integrated Nutrient Management for Potato-Maize-T. Aman Rice Cropping Pattern in Bangladesh. Rice J. 20 (1): 51-58.

Yoseftabar, S. 2012. Effect of nitrogen and phosphorus fertilizer on growth and yield rice (Oryza sativa L.). Int. J. Agron. Plant Prod. 3(12): 579-584. 